\title{
The Moderating Effect of Work-Related Risk on the Relationship between Cognitive-trust in Supervisor and Proactive Behavior
}

\author{
Pei LIU ${ }^{1, a}$, Wan-Fen GUO ${ }^{2, b, *}$ \\ ${ }^{1}$ Graduate School of Business Administration, Kobe University, Kobe 657-8501, Japan \\ ${ }^{2}$ The International College, Xiamen University, Xiamen 361102, Fujian, China \\ aryutsuchikau@yahoo.co.jp, bguowanfen@xmu.edu.cn \\ ${ }^{*}$ Corresponding author
}

Keywords: Proactive behavior, Cognitive-trust, Work-related risk, Nonlinear moderating effect.

\begin{abstract}
In occupational and organizational context, proactive behavior has attracted much attention over last decade. This study aims to examine how work-related risk influences the relationship between cognitive-trust in the supervisor and proactive behavior, using the data collected from 301 Chinese and 475 Japanese employees. By quadratic regression model, we found a fact in both Chinese and Japanese samples that the relationship between cognitive trust in supervisor and proactive behavior under Low-RISK situation tended to be a monotonic increasing curve, whereas a U-shape curve under High-RISK situation.
\end{abstract}

\section{Introduction}

Today's market, technology, and employment environments become increasingly varied and uncertain. In such a situation, employees' proactive behavior (PB), defined as self-initiated, anticipatory action that aims to change and improve the situation or oneself [1], is important for the success of both business and their career.

In the organizational and occupational context, a key antecedent of employees' $\mathrm{PB}$ is their cognitive-trust (CT) in the immediate supervisor [2,3]. But little is known about how situational factors, such as work-related risk (RISK), moderate the relation between CT in supervisor and PB. As [4] points out, since thus moderating effect may be nonlinear, it is difficult to accurately predict them. The reason why CT tends to interact nonlinearly with RISK is that CT is essentially based on the calculation of benefit-cost ratio [4]. Empirical researches are required to clarify the interaction between CT and RISK. In this paper, we examine the moderating effect of RISK on the relationship between $\mathrm{CT}$ and PB.

\section{Hypothesis}

PB can be characterized by a high-risk, high-return approach. On the one hand, to carry out PB, as an extra-role behavior, employees need to sacrifice a lot of private time and resources, and to suffer from long-term stress. Moreover, the failure probability of PB tends to be high in general, because it is essential to tackle new problems. On the other hand, once employees succeed in elevating group performance by $\mathrm{PB}$, it will substantially improve the status quo of department/organization, and contribute to their career development.

In organizational and occupational context, whether the employees' PB can succeed in improving group performance depends greatly on the decisions and actions of their supervisor. For example, performing PB which will change the work system and productive ability of the workplace needs the supervisor's permission and support. And, it is necessary for the supervisor to incorporate the new abilities created by employees' PB into the current work system of workplace.

Therefore, whether employees have positive expectations for the decisions and actions of their supervisor in various management situations, and the willingness to be vulnerable to the possibility of disappointment, will largely influence their decision to take PB. According to [5], thus positive expectations and willingness to be vulnerable are basic components of the definition of trust. In this 
study, we focus on employees' cognitive-trust in their supervisor, defined as such positive expectations and willingness stem from the knowledge of the supervisor's traits such as competence, benevolence, and integrity $[2,3,6]$. The stronger the $\mathrm{CT}$ in supervisor, the more likely it is that fruitful results of $\mathrm{PB}$ are in prospect, so that the employee will put more effort into PB. Thus, CT in the supervisor is positively related to $\mathrm{PB}$.

However, the relationship between employees' PB and group performance is affected not only by supervisor's decisions and actions, but also by situational factors such as work-related risk (RISK). By "risk", we mean the expected value (size $\times$ subjective probability) of the loss brought about by PB. Hence, it is likely that the casual effect of CT on PB is moderated by work-related risk in environment. Thus, we propose:

Hypothesis. The positive relationship between CT in supervisor and PB is moderated by RISK.

\section{Method}

We surveyed Chinese and Japanese employees respectively. The translation between Chinese and Japanese versions of our questionnaire was checked by a linguist.

Sample and Procedure. First, the samples of Chinese employees $(n=301)$ were gathered from seven cities of China (via website). $48.2 \%$ of respondents were male and $51.8 \%$ were female. Their average age was 31.39 years $(S D=7.48)$, average job tenure was 6.25 years $(S D=7.31)$, and the average length of time having worked with the current supervisor was 4.43 years $(S D=5.02)$. In terms of education, $6.3 \%$ had high school or vocational degrees, $23.9 \%$ had junior college degrees, $39.9 \%$ had college degrees, and $29.9 \%$ had graduate degrees. In terms of post, $49.8 \%$ were general employees, $18.9 \%$ were low level managers, $17.3 \%$ were middle level managers, $6.3 \%$ were top level managers, and others (7.6\%).

Next, we collected the samples $(n=475)$ from Japanese employees (through a research company). $54.7 \%$ of respondents were male and $45.3 \%$ were female. Their average age was 38.64 years $(S D=6.55)$, average job tenure was 12.90 years $(S D=7.72)$, and the average length of time having worked with the current supervisor was 4.31 years $(S D=4.76)$. In terms of education, $25.5 \%$ had high school degrees, $22.7 \%$ had junior college or vocational degrees, $42.9 \%$ had college degrees, and $8.8 \%$ had graduate degrees. In terms of post, $44.8 \%$ were general employees, $27.2 \%$ were low level managers, $22.1 \%$ were middle level managers, and others $(5.9 \%)$.

Measures. A 7-point Likert scale was used for all study measures. Cognitive-trust (CT) in supervisor was measured with a 14-item short form of Cognitive-Trust Scale (CTS-S; [2, 3]), ranging from 1 ("strongly disagree") to 7 ("strongly agree"). An example item was "Given my supervisor's track record, I see no reason to doubt his/her competence and preparation for the job" (Chinese sample, $\alpha=.97$; Japanese sample, $\alpha=.97$ ).

Proactive behavior (PB) was measured using [7]'s 12-item Proactive Behavior Scale (PBS), ranging from 1 ("strongly disagree") to 7 ("strongly agree"). An example item was "I anticipate changes, and voluntarily propose the solutions in workplace" (Chinese sample, $\alpha=.93$; Japanese sample, $\alpha=.95)$.

Since we were unable to locate an appropriate measure of work-related risk (RISK), we developed a five-item measure (Chinese sample, $\alpha=.86$; Japanese sample, $\alpha=.85$ ), ranging from 1 ("very low") to 7 ("very high"):

How much possibility is there that you and/or your organization will suffer a great loss

(1) when your department's objectives have not been achieved;

(2) due to the failures of your supervisor;

(3) due to your own faults;

(4) due to the errors in your supervisor's judgments?

And, (5) Overall, how much possibility is there that you will face risks?

\section{Results}

Descriptive Statistics and Correlations. Table 1 reported means, standard deviations, and correlations of three variables. 
Table 1. Descriptive statistics and correlations

\begin{tabular}{|c|c|c|c|c|c|c|c|c|}
\hline \multirow[b]{2}{*}{ Variable } & \multicolumn{4}{|c|}{ Chinese sample $(n=301)$} & \multicolumn{4}{|c|}{ Japanese sample $(n=475)$} \\
\hline & $\mathrm{M}(S D)$ & PB & $\mathrm{CT}$ & RISK & $\mathrm{M}(S D)$ & PB & $\mathrm{CT}$ & RISK \\
\hline Proactive behavior (PB) & $4.88(.94)$ & - & & & $4.53(.85)$ & - & & \\
\hline Cognitive trust in supervisor (CT) & $4.70(1.35)$ & $.39 * * *$ & - & & $4.17(1.21)$ & $.22 * * *$ & - & \\
\hline Work-related risk (RISK) & $4.16(1.24)$ & $.17 * *$ & -.040 & - & $4.45(.99)$ & $.28 * * *$ & $.11 *$ & - \\
\hline
\end{tabular}

Note. $* * * p<.001, * * p<.01, * p<.05$

Step1: A Problem in Applying Linear Model. Our hypothesis predicted that the level of RISK will moderate the relationship between CT in the supervisor and PB. In the step 1, we tested this moderating effect by linear regression model.

As shown in table 2, the causal effect of CT on PB under High-RISK situation was weaker than under Low-RISK situation, in both Chinese and Japanese samples. Especially in Chinese sample, the effect of $\mathrm{CT}$ on $\mathrm{PB}$ under High-RISK situation was not significant $(\beta=.15, p=.087)$. As mentioned above, these results can be explained by a fact that the moderating effect in question is nonlinear. Hence using usual linear model to analyze such moderating effects may be problematic.

Table 2. Moderating Effect of RISK by Linear Regression Model

\begin{tabular}{|c|c|c|c|c|c|c|c|c|c|c|c|c|}
\hline & \multicolumn{6}{|c|}{ Chinese sample $(n=301)$} & \multicolumn{6}{|c|}{ Japanese sample $(n=475)$} \\
\hline & \multicolumn{3}{|c|}{ Low-RISK $(n=165)$} & \multicolumn{3}{|c|}{ High-RISK $(n=136)$} & \multicolumn{3}{|c|}{ Low-RISK $(n=243)$} & \multicolumn{3}{|c|}{ High-RISK $(n=232)$} \\
\hline & $b$ & $\beta$ & $t$ & $b$ & $\beta$ & $t$ & $b$ & $\beta$ & $t$ & $b$ & $\beta$ & $t$ \\
\hline \multicolumn{3}{|c|}{ Intercept $2.43^{* * *}$} & 4.97 & $3.83 * * *$ & & 6.28 & $1.96 * * *$ & & 4.11 & $3.75 * * *$ & & 6.91 \\
\hline Gender & -.05 & -.03 & -.46 & $.34 *$ & $.17 *$ & 2.07 & .16 & .10 & 1.53 & -.01 & -.01 & -.09 \\
\hline Age & .02 & .17 & 1.40 & .03 & .22 & 1.51 & $.02 *$ & $.17 *$ & 2.10 & .02 & .13 & 1.42 \\
\hline Education & -.01 & -.01 & -.16 & -.16 & -.15 & -1.77 & $.12 *$ & $.15^{*}$ & 2.27 & -.06 & -.07 & -1.06 \\
\hline Tenure with supervisor & -.02 & -.13 & -1.11 & $-.08 * * *$ & $-.41 * * *$ & -3.42 & .00 & .02 & .37 & .01 & .06 & .87 \\
\hline Tenure & .01 & .11 & .71 & .00 & -.01 & -.05 & -.01 & -.10 & -1.26 & -.01 & -.13 & -1.49 \\
\hline Post & -.06 & -.09 & -1.30 & .04 & .06 & .75 & $.13^{*}$ & $.14^{*}$ & 2.05 & $.12 *$ & $.14^{*}$ & 2.00 \\
\hline $\mathrm{CT}$ & $.41^{* * *}$ & $.60 * * *$ & 8.73 & .10 & .15 & 1.72 & $.20^{* * * *}$ & $.26^{* * * *}$ & 4.20 & $.11^{*}$ & $.17 *$ & 2.51 \\
\hline$R^{2}$ & \multicolumn{3}{|c|}{.35} & \multicolumn{3}{|c|}{.18} & \multicolumn{3}{|c|}{.17} & \multicolumn{3}{|c|}{.06} \\
\hline$F$ statistic $(d f)$ & \multicolumn{3}{|c|}{$12.29 * * *(7 ; 157)$} & \multicolumn{3}{|c|}{$3.97 * * *(7 ; 128)$} & \multicolumn{3}{|c|}{$6.77 * * *(7 ; 235)$} & \multicolumn{3}{|c|}{$2.04 *(7 ; 224)$} \\
\hline
\end{tabular}

Step 2: Using Quadratic Regression Curve Model. In the step 2, we tested the moderating effect of RISK by quadratic regression curve model (1).

$$
P B=b_{0}+b_{1} C T+b_{2} C T^{2}
$$

Figure 1 provided the scatter diagrams, the estimated quadratic curve, and the estimates of the coefficients $b$. The scatter diagrams in Fig. 1 revealed a fact that in both Chinese [a] and Japanese samples [b], the relationship between CT and PB under Low-RISK situation was a monotonic increasing curve, whereas a U-shape curve under High-RISK situation. It is obvious that for analyzing the causal effect of CT on PB under High-RISK situation, the linear model is not adequate.

The table [c] in Fig. 1 demonstrated that the estimates of the coefficients $b$ representing the causal effect of CT on PB under High-RISK situation were significant, in both Chinese and Japanese samples.

Step 3: Using Quadratic Regression Surface Model. In the finally step, we used quadratic regression surface model (2) to confirm the facts found in above steps, by handling the level of RISK as continuous variable.

$$
P B=b_{0}+b_{1} C T+b_{2} R I S K+b_{3} C T^{2}+b_{4} C T \times R I S K+b_{5} R I S K^{2}
$$


[a] Chinese sample $(n=301)$
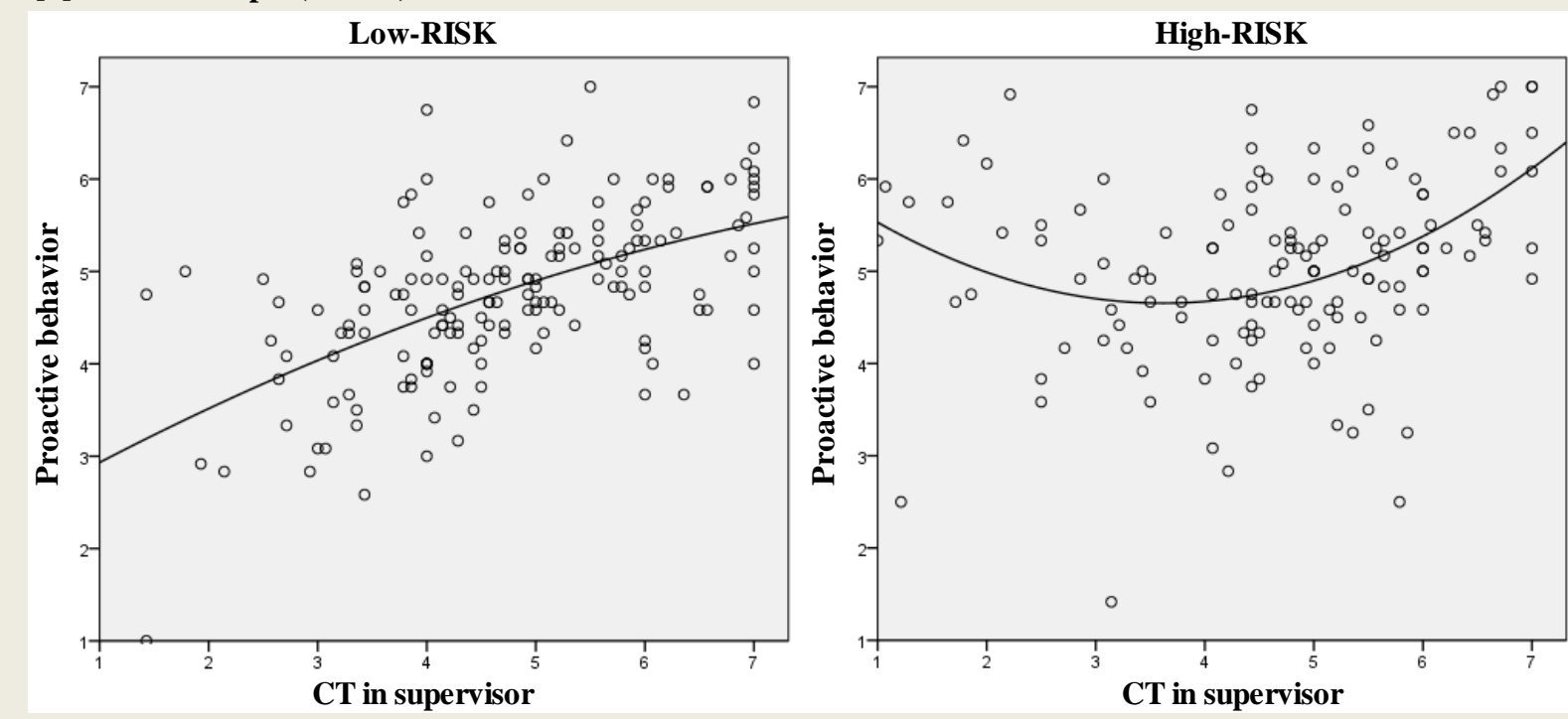

[b] Japanese sample $(n=475)$
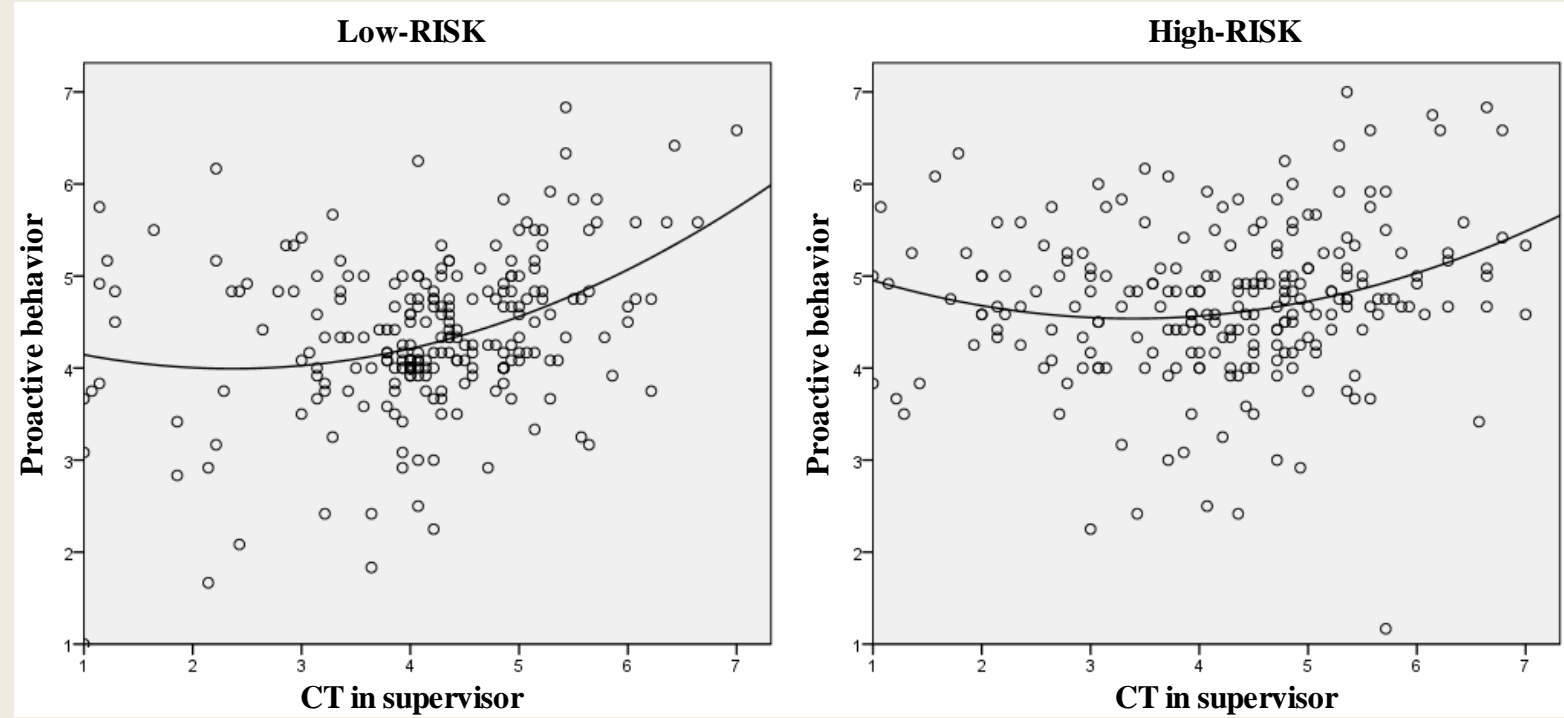

[c] Table: Estimation of Eq. 1

\begin{tabular}{|c|c|c|c|c|c|c|c|c|}
\hline & \multicolumn{4}{|c|}{ Chinese sample $(n=301)$} & \multicolumn{4}{|c|}{ Japanese sample $(n=475)$} \\
\hline & \multicolumn{2}{|c|}{ Low-RISK $(n=165)$} & \multicolumn{2}{|c|}{ High-RISK $(n=136)$} & \multicolumn{2}{|c|}{ Low-RISK $(n=243)$} & \multicolumn{2}{|c|}{ High-RISK $(n=232)$} \\
\hline & $b$ & $t$ & $b$ & $t$ & $b$ & $t$ & $b$ & $t$ \\
\hline Intercept & $2.29 * * *$ & 3.78 & $6.33 * * *$ & 11.22 & $4.45 * * *$ & 11.62 & $5.37 * * *$ & 13.76 \\
\hline $\mathrm{CT}$ & $.67 *$ & 2.55 & $-.92 * * *$ & -3.40 & -.38 & -1.93 & $-.49 *$ & -2.47 \\
\hline $\mathrm{CT}^{2}$ & -.03 & -1.09 & $.13 * * *$ & 4.08 & $.08 * *$ & 3.17 & $.07 * *$ & 2.97 \\
\hline$R^{2}$ & \multicolumn{2}{|c|}{.33} & \multicolumn{2}{|c|}{.16} & \multicolumn{2}{|c|}{.13} & \multicolumn{2}{|c|}{.06} \\
\hline$F$ statistic $(d f)$ & \multicolumn{2}{|c|}{$39.04 * * *(2 ; 162)$} & \multicolumn{2}{|c|}{$12.57 * * *(2 ; 133)$} & \multicolumn{2}{|c|}{$17.82 * * *(2 ; 240)$} & \multicolumn{2}{|c|}{$6.73 * * *(2 ; 229)$} \\
\hline
\end{tabular}

Note. ${ }^{* * *} p<.001, * * p<.01, * p<.05$

Figure 1. Moderating Effects of RISK by Quadratic Regression Curve Model

Fig. 2 provided the estimated quadratic regression surface and the estimates of the coefficients $b$. Looking at the graphs [a, b] in Fig. 2, we could grasp the overview of how the shape of relationship between CT and PB shifts from a monotonic increasing curve (almost linear) to a U-shape curve, as RISK increases from level 1 to 7.

On the basis of Eq. 2, we can express the causal effect of CT on PB in Eq. 3 


$$
\frac{\partial P B}{\partial C T}=b_{1}+2 b_{3} C T+b_{4} R I S K
$$

Which implies the causal effect $\partial P B / \partial C T$ is determined by the level of both CT and RISK. And in Eq. 3 , the coefficient $b_{4}$ represents the moderating effect of RISK on $\partial P B / \partial C T$. Table [c] in Fig. 2 showed that $b_{4}$ was significant in both Chinese $\left(b_{4}=-.08, p<.01\right)$ and Japanese samples $\left(b_{4}=-.07, p<\right.$ $.05)$, which directly supported our hypothesis.
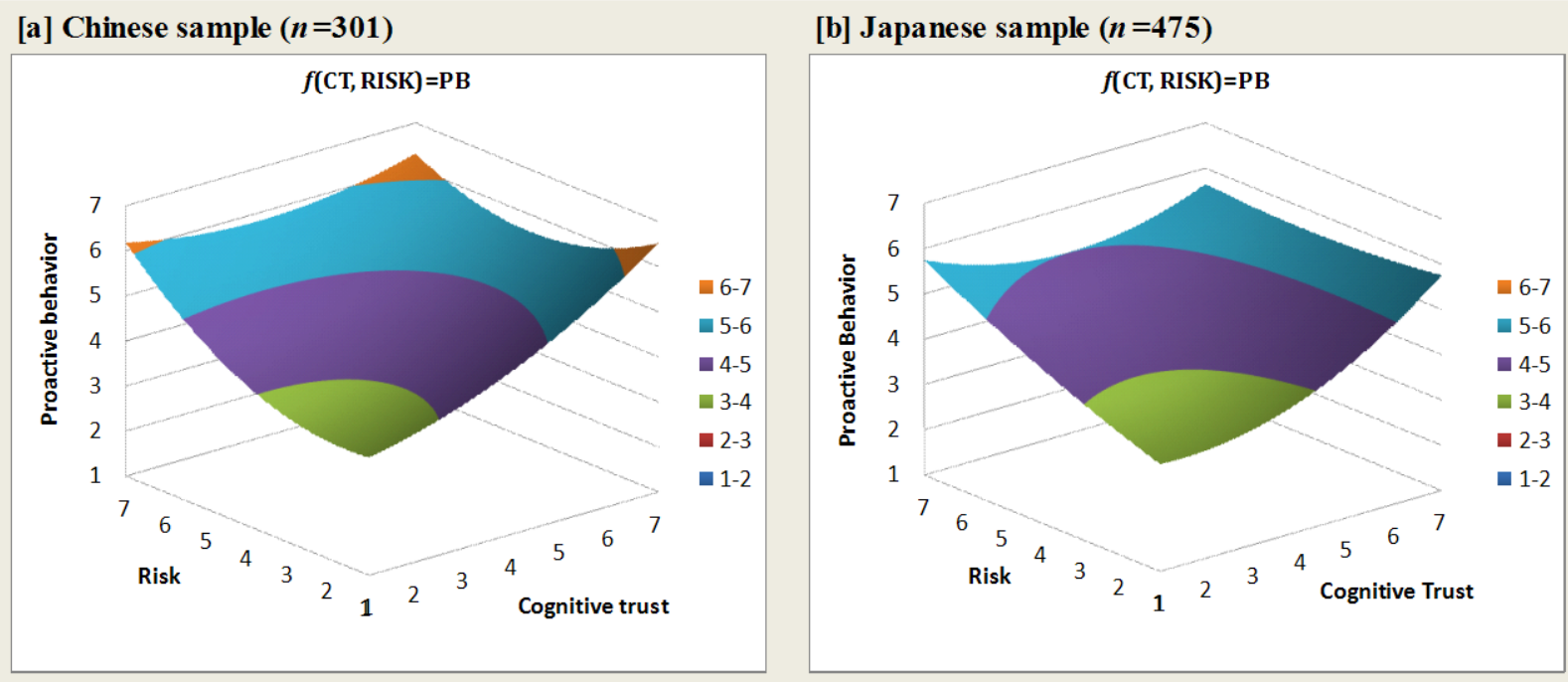

[c] Table: Estimation of Eq. 2

\begin{tabular}{|c|c|c|c|c|c|c|c|c|}
\hline & \multicolumn{4}{|c|}{ Chinese sample $(n=301)$} & \multicolumn{4}{|c|}{ Japanese sample $(n=475)$} \\
\hline & \multicolumn{2}{|c|}{ Linear model } & \multicolumn{2}{|c|}{ Quadratic model } & \multicolumn{2}{|c|}{ Linear model } & \multicolumn{2}{|c|}{ Quadratic model } \\
\hline & $b$ & $t$ & $b$ & $t$ & $b$ & $t$ & $b$ & $t$ \\
\hline Intercept $\left(b_{0}\right)$ & $3.00 * * *$ & 12.13 & $3.58 * * *$ & 4.69 & $2.98 * * *$ & 14.78 & $3.16 * * *$ & 5.23 \\
\hline $\mathrm{CT}\left(b_{1}\right)$ & $.28 * * *$ & 7.54 & .18 & .76 & $.13 * * *$ & 4.36 & -.15 & -.86 \\
\hline RISK $\left(b_{2}\right)$ & $.14 * * *$ & 3.59 & -.21 & -.95 & $.22 * * *$ & 5.96 & .33 & 1.42 \\
\hline $\mathrm{CT}^{2}\left(b_{3}\right)$ & - & - & $.05^{*}$ & 2.33 & - & - & $.08 * * *$ & 4.23 \\
\hline $\mathrm{CT} \times \operatorname{RISK}\left(b_{4}\right)$ & - & - & $-.08 * *$ & -2.97 & - & - & $-.07 *$ & -2.51 \\
\hline $\operatorname{RISK}^{2}\left(b_{5}\right)$ & - & - & $.09 * * *$ & 3.75 & - & - & .02 & .62 \\
\hline$R^{2}$ & \multicolumn{2}{|c|}{.19} & \multicolumn{2}{|c|}{.27} & \multicolumn{2}{|c|}{.11} & \multicolumn{2}{|c|}{.15} \\
\hline$F$ statistic $(d f)$ & \multicolumn{2}{|c|}{$33.88 * * *(2 ; 298)$} & \multicolumn{2}{|c|}{$21.50 * * *(5 ; 295)$} & \multicolumn{2}{|c|}{$30.34 * * *(2 ; 472)$} & \multicolumn{2}{|c|}{$16.82 * * *(5 ; 469)$} \\
\hline$\Delta R^{2}$ & & & \multicolumn{2}{|c|}{.08} & & & \multicolumn{2}{|c|}{.04} \\
\hline$\Delta F(d f)$ & & & \multicolumn{2}{|c|}{$10.98 * * *(3 ; 295)$} & & & \multicolumn{2}{|c|}{$7.02 * * *(3 ; 469)$} \\
\hline
\end{tabular}

Note. ${ }^{* * *} p<.001,{ }^{* *} p<.01,{ }^{*} p<.05$

Figure 2. Moderating Effects of RISK by Quadratic Regression Surface Model

\section{Discussion}

The Moderating Effect of RISK on the Relation between CT and PB. The purpose of this study is to examine how RISK influences the relationship between CT and PB. Our hypothesis predicted that the causal effect of CT in supervisor on PB is moderated by the level of RISK. The hypothesis was supported by both Chinese and Japanese samples, using quadratic regression surface model (2).

Theoretical Implication: An Explanation for the Nonlinear Moderating Effect of RISK. By quadratic regression model (1) and (2), we found a fact the relationship between CT in supervisor and PB under Low-RISK situation was a monotonic increasing curve, whereas a U-shape curve under High-RISK situation (Fig. 1, Fig. 2). This nonlinear pattern can be explained using the rationale of benefit-cost ratio. 
In Low-RISK situation, the success of employee's PB is mainly affected by the supervisor. The higher the supervisor's abillity, the more he/she can support the proactive empolyee. And the more the supervisor's benevolence, the more likely he/she wants to suport the proactive empolyee. Therefore the stonger the empolyee trusts in the supervisor, the higher the perceived potential benefits of PB.

However, in High-RISK situation, the employee is subjected to external pressure to tackle the problem. So when he/she does not trust in the supervisor, he/she has to engage in PB to make up for supervisor's inadequacies. Thus necessity of PB is relatively low when the employee moderately trusts in the supervisor, because he/she can tackle the problem in cooperation with the supervisor. When the employee strongly trust in the supervisor, he/she may put more effort into PB for the perceived potential benefits rather than for tackling the problem.

In conclusion, our research clarified how RISK moderates the effect of CT in supevisor on PB. We urge future research to examine more antecedents and moderators of employees' $\mathrm{PB}$, so that a deep understanding of how to promote PB can be gained.

\section{References}

[1] S. K. Parker, C. G. Collins, Taking stock: Integrating and differentiating multiple proactive behaviors, Journal of Management. 36 (2010)633-662.

[2] L. Pei, A theory on the functionally complementing interaction between cognitive- and emotional-trust: With an empirical research using the data of Japanese and Chinese employees, Kobe University Graduate Student Working Paper No. 201405a. (2014a)

http://www.b.kobe-u.ac.jp/stuwp/2014/201405a.pdf

[3] L. Pei, The influence process of cognitive- and emotional-trust in supervisor on employees' proactive behavior: A multiple-group analysis of Japanese and Chinese employees based on SEM approach, Kobe University Graduate Student Working Paper No. 201406a. (2014b) http://www.b.kobe-u.ac.jp/stuwp/2014/201406a.pdf

[4] K. Y. Ng, R. Y. J. Chua, Do I contribute more when I trust more? Differential effects of cognition- and affect-based trust, Management and Organization Review. 2 (2006)43-66.

[5] D. Rousseau, S. Sitkin, R. Burt, and C. Camerer, Not so different after all: A cross-discipline view of trust, Academy of Management Review. 23(1998)387-392.

[6] R. C. Mayer, J. H. Davis, F. D. Schoorman, An integrative model of organizational trust, Academy of Management Review. 20 (1995)709-734.

[7] L. Pei, The dimensional structure, scale of proactive behavior and comparison between Japan and China: Item response theory and structural equation modeling approach, Kobe University

Graduate Student Working Paper No. 201304a. (2013)

http://www.b.kobe-u.ac.jp/stuwp/2013/201304a.pdf 